\title{
Estimation of communication distance for VHF radios in Rayleigh channel
}

\author{
Li Jingwei ${ }^{\text {a }}$,QuanHoude and Cui Peizhang \\ Dept. of Information Engineering, Mechanical Engineering College, \\ Heping West Road No. 97, Shijiazhuang, Hebei, China \\ ajxyljw@163.com
}

\begin{abstract}
As a key indicator, communication distance directly affects the layout and the management of radios. In addition to the influence of the electrical performances, the radio is mainly affected by the channel and the background noise conditions. On the basis of the relationship of the propagation loss characteristic and the radio's performance, this paper obtains the relationship between the communication distance and the characteristics of Rayleigh channel. Simulation results show that the radio working on the lower frequency is effected more obviously by Rayleigh channel.

Keywords:communication distance; VHF radio; Rayleigh channel; BER; SNR; 2FSK system
\end{abstract}

\section{Introduction}

Very high frequency(VHF) radio has the characteristics of the transmission stability and large capacity, andis always used in vehicle-mounted communication. The communication distance is the key indicator of the radio, it directly effects the radio's performance $^{[1]}$. So estimating the communication distance has great practical significance $^{[2]}$.

VHF radios always work in the low band (30-88MHz), and the line-of-sight (LOS) propagation form. At the same time, the propagation lossis effected by the conductive of the ground. The area around the receiver, there are many multipath signals generated by the buildings, vehicles, trees, etc. The slow fading and the fast fading of the received signal are effected by propagation loss and multipath respectively.

Communication must conform with the bit error rate (BER) indicator, and the BER is related to the distance. This paper researches the relationship between the sending and receiving distance and the Rayleigh multiple channel about 2FSK wireless communication system. And the relationship results are shown by closed formula and simulation experiment.

This paper is organized as follows. Section 2 presents the Hata propagation loss model. Section 3 demonstrates the FSK systems performance formula in 
Rayleigh channels. Simulations results are in the section 4. Finally, section 5 presents some conclusions.

\section{Propagation Model}

Wireless signal after Rayleigh fading and additive Gaussian white noise interference, reaches the receiver, and the received signal is

$$
r(t)=\alpha(t) s(t)+n(t),
$$

where $s(t)$ is the transmitting signal, $\alpha(t)$ is the channel fading factor, $\alpha(t)=\zeta \times \xi(t), \zeta$ is the channel propagation loss, $\xi(t)$ is the Rayleigh fading process, $n(t)$ is the Additive White Gaussian Noise(AWGN). So Eq.1 can be rewritten as

$$
r(t)=\zeta \times \xi(t) \times s(t)+n(t)
$$

The characteristic of VHF communications. The VHF frequency band is defined as the frequency range from 30 to $300 \mathrm{MHz}$, and VHF wavelengths vary from 10-meters to 1-meter. This means that the size of antennas and tuning components used in VHF radio are much smaller and lighter than those of HF radios.

VHF radio wave has different propagation characteristic from HF radio wave. VHF radio frequency propagate principally along LOS paths. For the most part, the transmitting and receiving antennas must have a fairly unobstructed path between them for communication to take place.

Hata propagation loss model.There are a number of factors affecting the path loss in mobile communications. Among these factors are base-station antenna height, mobile antenna height, operating frequency, atmospheric conditions, and presence or absence of buildings and trees. Various mean path loss models have been developed that incorporate such factors.

In an attempt to make the Okumura's model easier for computer implementation Hata has fit Okumura's cures with analytical expressions ${ }^{[3,4]}$. This makes the computer implementation of the model straightforward. Hata's formulation is limited to some values of input parameters.

Hata's model for prediction and the range of parameters for its applicability is given as:

$$
\begin{aligned}
& L_{\text {Hata }}=10 \log \left(\frac{P_{t} G_{t}}{P_{r}}\right)=69.55+26.16 \log f-13.82 \log \left(\frac{h_{t}}{h_{0}}\right)+a\left(h_{r}\right) \\
& +\left(44.9-6.55 \log \left(\frac{h_{t}}{h_{0}}\right)\right) \log \left(\frac{R}{R_{0}}\right)-A_{a}+D L,
\end{aligned}
$$


where $P_{r}$ is received signal level in $\mathrm{dBm}, P_{t}$ is transmitted power in $\mathrm{dBm}, G_{t}$ is transmit antenna gain in the direction of the receiver in $\mathrm{dB}, f$ is operating frequency in $\mathrm{MHz}, h_{t}$ is effective transmitted antenna height in $\mathrm{m}, h_{0}$ is reference transmitted antenna height, selected as $1 \mathrm{~m}, h_{r}$ is received antenna height in $\mathrm{m}$, $a\left(h_{r}\right)$ is received antenna height correction in $\mathrm{dB}, R$ is distance between the receiver and the transmitter in $\mathrm{km}, R_{0}$ is reference distance, selected $1 \mathrm{~km}, D L$ is diffraction losses in $\mathrm{dB}, A_{a}$ is area adjustment factor in $\mathrm{dB}$.

The received antenna height correction factor is computed as: For a small and medium size city,

$$
a\left(h_{r}\right)=(1.1 \log f-0.7) h_{r}-(1.56 \log f-0.8) .
$$

For a large city,

$$
\begin{aligned}
& a\left(h_{r}\right)=8.29\left(\log \left(1.54 h_{r}\right)\right)^{2}-1.1 f \leq 200 \mathrm{MHz} . \\
& a\left(h_{r}\right)=3.2\left(\log \left(11.75 h_{r}\right)\right)^{2}-4.97 f \geq 400 \mathrm{MHz} .
\end{aligned}
$$

The area correction factor can be computed as: For suburban areas,

$$
A_{a}=5.4+2 \times\left(\log \frac{f}{28}\right)^{2} \mathrm{~dB} \text {. }
$$

For open areas,

$$
A_{a}=4.78(\log f)^{2}-18.33 \log f+40.94 \mathrm{~dB} .
$$

\section{Error Probability in Rayleigh Channel Model}

Let assume that the multipath components are not resolvable, so the channel is frequency nonselective. If we don't regard the channel propagation loss temporarily, the channel impulse response is represented as

$$
h(\tau: t)=\xi \times \delta\left(\tau-\tau_{0}\right),
$$

where the amplitude $\xi$ is Rayleigh distributed; i.e.,

$$
f(\xi)=\left\{\begin{array}{ll}
\frac{\xi}{\sigma^{2}} e^{-\xi^{2} / 2 \sigma^{2}}, & \xi>0 \\
0, & \xi \leq 0
\end{array} .\right.
$$


If the binary signals are orthogonal, as in non-coherent FSK, where the two possible transmitted signals are given as

$$
r(t)=\xi \sqrt{\frac{2 \varepsilon_{b}}{T}} \cos \left[2 \pi\left(f_{c}+\frac{m}{2 T}\right) t+\phi\right]+n(t) \quad m=0,1 .
$$

The received signal is

$$
u_{m}(t)=\sqrt{\frac{2 \varepsilon_{b}}{N_{0}}} \cos \left[2 \pi\left(f_{c}+\frac{m}{2 T}\right) t\right]
$$

In this case, the received signal is cross non-correlated with the two signals

$$
\begin{aligned}
& \Psi_{1}(t)=\sqrt{\frac{2}{T}} \cos \left(2 \pi f_{c} t+\phi\right), \\
& \Psi_{2}(t)=\sqrt{\frac{2}{T}} \cos \left(2 \pi\left(f_{c}+\frac{1}{2 T}\right) t+\phi\right) .
\end{aligned}
$$

If $m=0$, for example, the two non-correlated outputs are

$$
r_{1}=\alpha \sqrt{\varepsilon_{b}}+n_{1}
$$

$$
r_{2}=n_{2}
$$

where $n_{1}$ and $n_{2}$ are the additive noise components at the outputs of the two non-correlated signals. Hence, the probability of error is simply the probability that $r_{2}>r_{1}$. Since the signals are orthogonal, the probability of error for a fixed valued of $\alpha$ has the familiar form ${ }^{[5,6]}$

$$
P_{b}(\alpha)=\frac{1}{2} e^{-\rho_{b} / 2} \text {. }
$$

We view $P_{b}(\alpha)$ as a conditional probability of error for a given value of the channel attenuation $\xi$. To determine the probability of error averaged over all possible values of $\xi$, we compute the integral

$$
P_{b}=\int_{0}^{\infty} P_{b}(\xi) f(\xi) d \xi,
$$


where $f(\xi)$ is the Rayleigh PDF given by Eq.10. This integral has the simple closed form expression

$$
P_{b}=\frac{1}{2+\bar{\rho}_{b}},
$$

where, $\bar{\rho}_{b}$ by definition,

$$
\bar{\rho}_{b}=\frac{\varepsilon_{b}}{N_{0}} E\left(\xi^{2}\right)
$$

Hence, $\bar{\rho}_{b}$ is the average received SNR/bit and $E\left(\xi^{2}\right)=2 \sigma^{2}$. Thus, when $\bar{\rho}_{b} \square 1$, Eq.19 simplify to

$$
P_{b} \approx \frac{1}{\bar{\rho}_{b}}=\frac{N_{0}}{2 \varepsilon_{b} \sigma^{2}}
$$

\section{The Estimation for Communication Distance}

Through above analysis, the communication distance is effected by transmitted power, operating frequency, antennas height, geographical conditions and so on. Of course, channel fading and background noise affect the SNR, and then affect BER. We can see that there is a certain relationship between communication distance and BER.

The relationship between communication distance and Rayleigh channel's parameter.From Eq.21, we can obtain signal's energy

$$
\varepsilon_{b}=\frac{N_{0}}{2 P_{b} \sigma^{2}} \text {. }
$$

At the receiving end, from Eq.3, we can obtain the received signal energy

$$
P_{r}=P_{t} G_{t} 10^{-L_{\text {Hata }} / 10} \text {. }
$$

Eq.22 equals Eq.23, we can obtain propagation loss $L_{\text {Hata }}$ and the Rayleigh channel parameter $\sigma^{2}$

$$
L_{\text {Hata }}=-10 \log \frac{N_{0}}{2 P_{t} G_{t} P_{b} \sigma^{2}} .
$$


Eq.3 equals Eq.24, the relationship between the Rayleigh channel parameter $\sigma^{2}$ and the communication distance $R$ is

$$
\log R=\frac{-10 \log \frac{N_{0}}{2 P_{t} G_{t} P_{b} \sigma^{2}}-L_{0}}{L_{R}},
$$

where $R$ is the communication distance, $N_{0}$ is the noise power, $P_{t}$ is the transmitted power, $G_{t}$ is the transmitted gain, $P_{b}$ is the BER of the wireless system, $\sigma^{2}$ is the parameter of Rayleigh channel, $L_{0}$ and $L_{R}$ equal respectively

$$
\begin{aligned}
& L_{0}=69.55+26.16 \log f-13.82 \log h_{t}+a\left(h_{r}\right)-A_{a}+D L, \\
& L_{r}=44.9-6.55 \log h_{t},
\end{aligned}
$$

The meaning of the parameters in the Eq.26 and the Eq.27 are the same meaning as them in the Eq.3.

Simulation experiment and analysis.In order to verify the influence of Rayleigh channel on the communication distance, we have made simulation on the relationship between both of them.

The simulation experimental parameters assumed that: the operating frequency is $68 \mathrm{MHz}, 98 \mathrm{MHz}$, and $128 \mathrm{MHz}$ respectively, the antenna heights of transmitting and receiving are $6 \mathrm{~m}$, transmitting power is $20 \mathrm{w}$, transmitting gain is $0 \mathrm{~dB}$, the BER of receiver is $10^{-4}$, the background AGWN power is $10^{-13} \mathrm{~mW}$.

The simulation results in the suburbs of small city are shown in Fig. 1. We can see that the communication distance gradually get farther with the increase of the parameter of Rayleigh channel, and closer with inverse of operating frequency. We also see that in the low frequencies the communication distances have bigger change than the high frequency. That is to say the influence of Rayleigh channel is more obviously on lower frequency communication than higher frequency communication. 


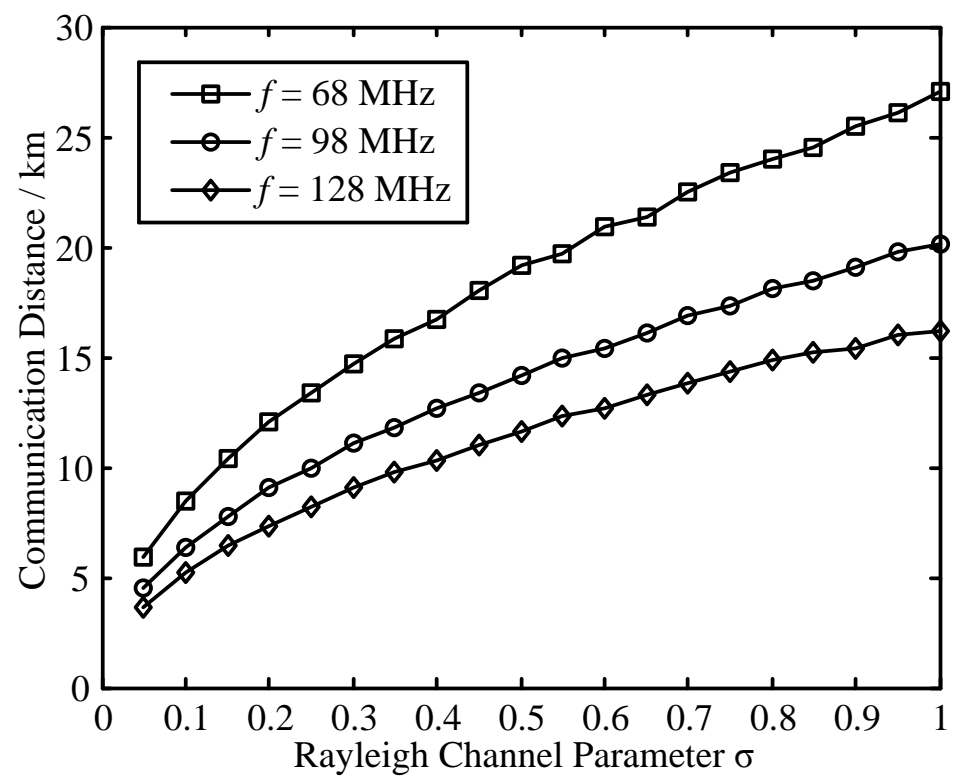

Fig. 1. The communication distance effected by Rayleigh channel

\section{Conclusions}

Through analysis, the communication distance is affected by theelectrical properties ofradios, the wireless channels and background noise. We find the relationship between communication distance and Rayleigh channels parameter based on the receiving power. And then we research this relationship through computer simulation. Results show the Rayleigh channel has more influence on low frequency. This study has a certain guiding significance to evaluate the performance of radios.

\section{References}

[1]S. Sathyamurthy and S Sundaredh: Defence Science Journal. Vol. 58(2008), p. $762-767$

[2] J. Honda, K. Uchida and K. Yoon: IEICE Transactions on Electronics. Vol. 1(2010), p. 39-45

[3] M. Hatay:IEEE Transactions on Vehicular Technology. Vol. 29(1999), p. 317-325

[4] S. Jiang and S. Georgakopoulos: Journal of Electromagnetic Analysis and Application. Vol. 3(2011), p. 261-266

[5] Q. T. Zhang: IEEE Transactions on Communications. Vol. 45(1997), p.270-273 
[6] J. G. Proakis and M. Salehi: Digital Communications (McGraw-Hill, USA 2008) 